\section{BLOOD GAS MANAGEMENT AND DEGREE OF COOLING: EFFECTS ON CEREBRAL METABOLISM BEFORE AND AFTER CIRCULATORY ARREST}

This study investigated the effects of different cooling strategies on cerebral metabolic response to circulatory arrest. In particular, it examined the impact of blood gas management and degree of cooling on cerebral metabolism before and after deep hypothermic circulatory arrest. Sixty-nine 1-week-old piglets $\left(2\right.$ to $3 \mathrm{~kg}$ ) were placed on cardiopulmonary bypass $\left(37^{\circ} \mathrm{C}\right.$ ) at $100 \mathrm{ml} / \mathrm{kg}$ per minute. Animals were cooled to $18^{\circ}$ or $14^{\circ} \mathrm{C}$ as follows: alpha-stat strategy to $18^{\circ} \mathrm{C}(n=9)$ or $14^{\circ} \mathrm{C}(n=6)$, pH-stat strategy to $18^{\circ} \mathrm{C}(n=9)$ or $14^{\circ} \mathrm{C}(n=$ 7), or pH-stat strategy for 18 minutes followed by a switch to alpha-stat strategy for the last 5 minutes of cooling to $18^{\circ} \mathrm{C}(n=12)$ or $14^{\circ} \mathrm{C}(n=10)$. Animals underwent 60 minutes of circulatory arrest followed by rewarming with alpha-stat strategy to $36^{\circ} \mathrm{C}$. Control animals were cooled with alpha-stat strategy to $18^{\circ} \mathrm{C}(n=10)$ or $14^{\circ} \mathrm{C}(n=3)$ and then maintained on cold cardiopulmonary bypass $(100 \mathrm{ml} / \mathrm{kg}$ per minute) for 60 minutes. Three animals were excluded (see text). With the use of xenon 133 clearance methods, cerebral blood flow was measured at the following points: point $I$, cardiopulmonary bypass $\left(37^{\circ} \mathrm{C}\right)$; point $\mathrm{II}$, cardiopulmonary bypass before circulatory arrest or control flow $\left(18^{\circ}\right.$ or $\left.14^{\circ} \mathrm{C}\right)$; and point III, cardiopulmonary bypass after rewarming $\left(36^{\circ} \mathrm{C}\right)$. Cerebral metabolic rate of oxygen consumption was calculated for each point. At point II, cerebral metabolism was more suppressed at $14^{\circ} \mathrm{C}$ compared with that at $18^{\circ} \mathrm{C}$. At any given temperature $\left(18^{\circ}\right.$ or $\left.14^{\circ} \mathrm{C}\right)$, pH-stat strategy provided the greatest suppression of cerebral metabolism. In control animals, cerebral metabolic oxygen consumption at point III returned to baseline values after 60 minutes of cold bypass. Sixty minutes of circulatory arrest resulted in a significant reduction in cerebral metabolic oxygen consumption at point III compared with that at point I regardless of cooling temperature or blood gas strategy. The amount of cerebral metabolic recovery was significantly reduced in the pH-stat $14^{\circ} \mathrm{C}$ group compared with that in the pH-stat $18^{\circ} \mathrm{C}$ group at point III. The use of $\mathrm{pH}$-stat strategy followed by a switch to alpha-stat at $14^{\circ} \mathrm{C}$ provided better cerebral metabolic recovery compared with either strategy used alone. The use of $\mathrm{pH}$-stat strategy during initial cooling may provide the animal with maximal cerebral metabolic suppression. The cerebral acidosis produced with pH-stat cooling may worsen cerebral metabolic injury from circulatory arrest, but this effect is eliminated with the use of alpha-stat just before the period of circulatory arrest. (J THORaC Cardiovasc SuRg 1995;110:1649-57)

Lynne A. Skaryak, MD, ${ }^{a}$ Paul J. Chai, MD $_{9}{ }^{a}$ Frank H. Kern, MD, ${ }^{b}$

William J. Greeley, MD, ${ }^{b}$ and Ross M. Ungerleider, MD, ${ }^{a}$ Durham, N.C.
From the Departments of Surgery, ${ }^{\text {a }}$ Anesthesiology, and Pediatrics, ${ }^{b}$ Duke University Medical Center, Durham, N.C.

Supported in part by The Children's Miracle Network Telethon.

Presented in part at The World Congress of Pediatric Cardiology and Cardiac Surgery, Paris, France, June 21-25, 1993, and at The American Heart Association, Sixty-sixth Scientific Sessions, Atlanta, Ga., November 8-11, 1993.
Received for publication Sept. 15, 1994.

Accepted for publication Feb. 27, 1995.

Address for reprints: Ross M. Ungerleider, MD, Department of Surgery, Box 3178, Duke University Medical Center, Durham, NC 27710.

Copyright (C) 1995 by Mosby-Year Book, Inc.

$0022-5223 / 95 \$ 5.00+0 \quad 12 / 1 / 64508$ 
D eep hypothermic circulatory arrest (DHCA) is often used during the repair of complex congenital heart disease in neonates. Transient and permanent neuropsychologic injury may be observed after DHCA. ${ }^{1-3}$ The optimal goal during active cooling before a period of DHCA is to uniformly deliver cold perfusate to the brain so that there is a homogeneous reduction in temperature and metabolic activity. The reduction of cerebral metabolic rate of oxygen consumption $\left(\mathrm{CMRO}_{2}\right)$ at the start of DHCA is crucial for maintenance of energy stores and organ protection. ${ }^{4}$

Strategies during the cooling phase that would result in the lowest $\mathrm{CMRO}_{2}$ before DHCA may provide the best cerebral protection. Two such strategies are the use of more profound hypothermia and the use of pH-stat blood gas management. Because $\mathrm{CMRO}_{2}$ is exponentially related to temperature during hypothermic cardiopulmonary bypass $(\mathrm{CPB}){ }^{5}$ the use of more profound hypothermia during cooling may provide better global suppression of $\mathrm{CMRO}_{2}$ before DHCA. Alpha-stat and $\mathrm{pH}$-stat are blood gas management strategies used to regulate arterial carbon dioxide tension $\left(\mathrm{PaCO}_{2}\right)$ during hypothermia. Alpha-stat strategy maintains a $\mathrm{PaCO}_{2}$ level of $40 \mathrm{~mm} \mathrm{Hg}$ when measured by the gas analyzer at $37^{\circ} \mathrm{C}$ (temperature uncorrected). The $\mathrm{pH}$-stat strategy uses the addition of carbon dioxide to the oxygenator gas flow during cooling to achieve a $\mathrm{PaCO}_{2}$ level of $40 \mathrm{~mm} \mathrm{Hg}$ when corrected to the patient's core temperature (temperature corrected). This addition of carbon dioxide causes increased cerebral blood flow (CBF) during hypothermic $\mathrm{CPB}^{6-9}$ The advantages of the use of alpha-stat when compared with $\mathrm{pH}$-stat management are more optimal buffering capacity and enzymatic function, ${ }^{10}$ preservation of flow metabolism coupling, ${ }^{6}$ and reduction in progressive tissue acidosis during and after a period of DHCA. ${ }^{11}$ The advantages of alphastat strategy may be outweighed by less effective cooling of the brain and therefore less suppression of cerebral metabolism at the start of ischemia.

This study examined the effects of blood gas management (alpha-stat and $\mathrm{pH}$-stat) and degree of cooling $\left(14^{\circ}\right.$ and $\left.18^{\circ} \mathrm{C}\right)$ on cerebral metabolism before and after a 60 -minute period of DHCA. The techniques used in this investigation were designed to duplicate those used in the clinical setting.

\section{Methods}

Animal preparation. Sixty-nine 1-week-old piglets weighing 2 to $3 \mathrm{~kg}$ were studied with approval of the institution's
Animal Care and Use Committee and in compliance with the "Guide for the Care and Use of Laboratory Animals" published by the National Institutes of Health $(\mathrm{NIH}$ Publication No. 85-23, revised 1985). Animals were premedicated with intramuscular ketamine $(20 \mathrm{mg} / \mathrm{kg})$ and intubated and the lungs mechanically ventilated (infant ventilator; Sechrist Industries, Anaheim, Calif.). Methylprednisolone $(40 \mathrm{mg} / \mathrm{kg})$ was given intravenously. After a bolus of intravenous fentanyl $(100 \mu \mathrm{g})$ and pancuronium $(0.1 \mathrm{mg} / \mathrm{kg})$, anesthesia was maintained with continuous fentanyl infusion $\left(50 \mu \mathrm{g} \cdot \mathrm{kg}^{-1} \cdot \mathrm{hr}^{-1}\right)$. A femoral artery catheter was placed for measurement of mean arterial pressure and arterial blood gas sampling. Temperature probes were placed in the nasopharynx and cortical region of the brain and a median sternotomy was performed. A burr hole was made in the skull at the crus of the sagittal and coronal sutures to allow sagittal sinus blood sampling.

Method of CBF measurement. Hemispheric CBF was measured by the xenon $\left({ }^{133} \mathrm{Xe}\right)$ clearance technique previously described by this group and others. ${ }^{12,13}$ Two extracranial $16 \mathrm{~mm}$ cadmium telluride gamma emission detectors were placed over the right and left temporal lobes. Radioactive xenon $(1.5 \mathrm{mCi}$ in $2 \mathrm{ml}$ of normal saline solution) was injected into the aortic cannula and radioactive decay recorded from each hemisphere over a 5 -minute period. With use of a modification of the initial slope index method, CBF was determined. ${ }^{14}$ By this method, $\mathrm{CBF}=($ slope $)(\lambda)(100)$, where slope is the natural logarithm of the ${ }^{133} \mathrm{Xe}$ clearance 1 minute after the peak of the curve, $\lambda$ is the tissue-blood partition coefficient for xenon corrected for hematocrit and temperature, ${ }^{15}$ and 100 converts milliliters times grams ${ }^{-1}$ times minutes ${ }^{-1}$ to milliliters times $100 \mathrm{gm}^{-1}$ times minutes ${ }^{-1}$. Global CBF was determined from the average of the individually calculated hemispheric blood flow values. The baseline ${ }^{133} \mathrm{Xe}$ value was measured before each determination and subtracted to correct curves for residual xenon. This method has recently been validated during hypothermic CPB. ${ }^{13}$

Perfusion technique. A Stöckert-Shiley (Irvine, Calif.) Computer Assisted Perfusion System nonpulsatile roller pump and a Cobe VP-CML (Denver, Colo.) membrane oxygenator with venous reservoir comprised the CPB circuit. Circuit blood gas values were monitored with a CDI-300 (CDI; 3M Healthcare, Irvine, Calif.) continuous in-line blood gas analyzer. The circuit was primed with fresh heparinized donor pig whole blood with a hematocrit value of $26 \% \pm 0.3 \%$. After animals were heparinized $(500 \mathrm{U} / \mathrm{kg})$, an $8 \mathrm{~F}$ arterial and a $14 \mathrm{~F}$ venous cannula were inserted in the ascending aorta and right atrium, respectively, and CPB initiated. Pump flow was maintained at a constant flow rate of $100 \mathrm{ml} \cdot \mathrm{kg}^{-1} \cdot \mathrm{min}^{-1}$ throughout the duration of CPB. Pharmacologic agents were not administered to control blood pressure during CPB. Sodium bicarbonate was given to maintain a base excess between -3 and $3 \mathrm{mmol} / \mathrm{L}$. The $\mathrm{PaCO}_{2}$ was maintained at a predetermined level according to the study protocol by adjusting the gas flow rate in the oxygenator with the addition of 5\% $\mathrm{CO}_{2}$ as necessary during the period of hypothermic CPB.

Study protocol. A schematic diagram of the experimental protocol and data collection sequence is shown in Fig. 1. After instrumentation, animals were placed on $\mathrm{CPB}$ 
$\left(37.2^{\circ} \pm 0.1^{\circ} \mathrm{C}\right)$ at $100 \mathrm{ml} / \mathrm{kg}$ per minute and baseline measurements (point I) were obtained. Animals were cooled to $18.4^{\circ} \pm 0.1^{\circ} \mathrm{C}$ or $14.0^{\circ} \pm 0.2^{\circ} \mathrm{C}$ over 23 minutes and data (point II) were obtained. The experimental groups were cooled as follows: the $\alpha\left(18^{\circ} \mathrm{C}\right)$ group received alpha-stat strategy to $18^{\circ} \mathrm{C}(n=9)$; the $\mathrm{pH}\left(18^{\circ} \mathrm{C}\right)$ group received $\mathrm{pH}$-stat strategy to $18^{\circ} \mathrm{C}(n=9)$; the $\mathrm{pH} \rightarrow \alpha\left(18^{\circ} \mathrm{C}\right)$ group received $\mathrm{pH}$-stat strategy for 18 minutes followed by a switch to alpha-stat strategy for the last 5 minutes of cooling to $18^{\circ} \mathrm{C}(n=12)$; the $\alpha\left(14^{\circ} \mathrm{C}\right)$ group received alpha-stat strategy to $14^{\circ} \mathrm{C}(n=6)$; the $\mathrm{pH}\left(14^{\circ} \mathrm{C}\right)$ group received $\mathrm{pH}$-stat strategy to $14^{\circ} \mathrm{C}(n=$ $7)$; and the $\mathrm{pH} \rightarrow \alpha\left(14^{\circ} \mathrm{C}\right)$ group received $\mathrm{pH}$-stat strategy for 18 minutes followed by a switch to alpha-stat strategy for the last 5 minutes of cooling to $14^{\circ} \mathrm{C}(n=10)$. Alpha-stat strategy was defined as the maintenance of $\mathrm{PaCO}_{2}$ at $40 \pm 5 \mathrm{~mm} \mathrm{Hg}$, temperature uncorrected, and pH-stat as maintenance of $\mathrm{PaCO}_{2}$ at $40 \pm 5 \mathrm{~mm} \mathrm{Hg}$, temperature corrected. Animals underwent 60 minutes of DHCA followed by rewarming with alpha-stat strategy to $36.4^{\circ} \pm 0.1^{\circ} \mathrm{C}$ over $26 \pm 1$ minutes and final measurements were obtained during CPB (point III). Not shown in this schematic are control animals that were cooled with alpha-stat strategy to $18^{\circ} \mathrm{C}$ (group CTL $\left[18^{\circ} \mathrm{C}\right], n=10$ ) or $14^{\circ} \mathrm{C}$ (group CTL $\left[14^{\circ} \mathrm{C}\right], n=3$ ) followed by 60 minutes of deep hypothermic CPB at $100 \mathrm{ml} / \mathrm{kg}$ per minute. The duration of CPB for animals undergoing DHCA was $84 \pm$ 1 minutes and $149 \pm 3$ minutes for those undergoing deep hypothermic CPB.

Nasopharyngeal and cortical brain temperatures, mean arterial pressure, arterial and sagittal sinus blood gas values, hemoglobin concentration, and $\mathrm{CBF}$ were measured at each stage. The cerebral arteriovenous oxygen content difference $\left(\mathrm{cCa}-\mathrm{vO}_{2}\right)$ was then calculated as $\mathrm{CaO}_{2}$ - $\mathrm{CssO}_{2}$, where $\mathrm{CaO}_{2}$ is the arterial oxygen content and $\mathrm{CsSO}_{2}$ is the sagittal sinus oxygen content. A GEM-STAT blood gas/electrolyte monitor (Mallinckrodt, Ann Arbor, Mich.) was used to measure blood gas values. Oxygen saturation and hemoglobin were measured by an IL 482 Co-Oximeter system (Instrumentation Laboratory, Lexington, Mass.). Cerebral metabolism $\left(\mathrm{CMRO}_{2}\right)$, cerebral oxygen delivery $\left(\mathrm{cD} \cdot \mathrm{O}_{2}\right.$ ), and cerebral oxygen extraction ratio $\left(\mathrm{CO}_{2} \mathrm{ER}\right)$ were calculated as follows:

$$
\begin{gathered}
\mathrm{CMRO}_{2}\left(\mathrm{ml} \cdot 100 \mathrm{gm} \mathrm{brain}^{-1} \cdot \mathrm{min}^{-1}\right)=\left(\mathrm{cCa}-\mathrm{vO}_{2}\right)(\mathrm{CBF}) \\
\mathrm{cD} \cdot \mathrm{O}_{2}\left(\mathrm{ml} \mathrm{O}_{2} \cdot 100 \mathrm{gm} \mathrm{brain}^{-1} \cdot \mathrm{min}^{-1}\right)=\left(\mathrm{CaO}_{2}\right)(\mathrm{CBF}) \\
\mathrm{CO}_{2} \mathrm{ER}(\%)=\mathrm{CMRO}_{2} / \mathrm{cD} \cdot \mathrm{O}_{2}
\end{gathered}
$$

Statistical analysis. Data were analyzed by the Student's paired $t$ test to compare results within each group. The unpaired $t$ test and analysis of variance were used to compare results between groups. All results are expressed as means plus or minus the standard errors. Significance was defined as $p<0.05$.

\section{Results}

The study was successfully completed in 66 of the 69 animals. Three animals were excluded, two be-

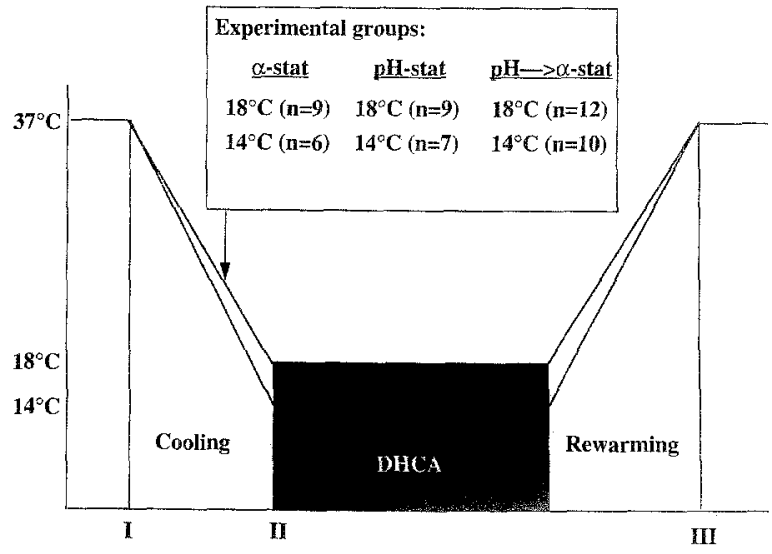

Fig. 1. Experimental protocol and data collection sequence. After instrumentation, animals were placed on CPB at $100 \mathrm{ml} / \mathrm{kg}$ per minute and $37^{\circ} \mathrm{C}$ and baseline measurements (I) were obtained. Animals were cooled to $18^{\circ}$ or $14^{\circ} \mathrm{C}$ over 23 minutes and data (II) were obtained. Experimental groups were cooled as follows: $\alpha\left(18^{\circ} \mathrm{C}\right)$, alpha-stat strategy to $18^{\circ} \mathrm{C}(n=9) ; \mathrm{pH}\left(18^{\circ} \mathrm{C}\right)$, pH-stat strategy to $18^{\circ} \mathrm{C}(n=9) ; \mathrm{pH} \rightarrow \alpha\left(18^{\circ} \mathrm{C}\right), \mathrm{pH}$-stat strategy for 18 minutes followed by switch to alpha-stat strategy for last 5 minutes of cooling to $18^{\circ} \mathrm{C}(n=12) ; \alpha\left(14^{\circ} \mathrm{C}\right)$, alpha-stat strategy to $14^{\circ} \mathrm{C}(n=6) ; \mathrm{pH}\left(14^{\circ} \mathrm{C}\right)$, pH-stat strategy to $14^{\circ} \mathrm{C}(n=7) ; \mathrm{pH} \rightarrow \alpha\left(14^{\circ} \mathrm{C}\right), \mathrm{pH}$-stat strategy for 18 minutes followed by switch to alpha-stat strategy for last 5 minutes of cooling to $14^{\circ} \mathrm{C}(n=10)$. Animals underwent 60 minutes of DHCA followed by rewarming to $36^{\circ} \mathrm{C}$ over 26 -minute period. Final measurements were obtained at this time (III). Not shown in this schematic are control animals, which were cooled with alpha-stat strategy to $18^{\circ} \mathrm{C}\left(\mathrm{CTL}\left[18^{\circ} \mathrm{C}\right.\right.$, $\left.n=10\right)$ or $14^{\circ} \mathrm{C}$ (CTL $\left[14^{\circ} \mathrm{C}\right.$, $n=3$ ) followed by 60 minutes of deep hypothermic $\mathrm{CPB}$ $(100 \mathrm{ml} / \mathrm{kg}$ per minute).

cause of markedly abnormal baseline $\mathrm{CMRO}_{2}$ values (groups $\mathrm{pH}\left[18^{\circ} \mathrm{C}\right]$ and $\alpha\left[14^{\circ} \mathrm{C}\right]$ ) and one because of the inability to adequately rewarm to a nasopharyngeal temperature of $36^{\circ} \mathrm{C}$ within $30 \mathrm{~min}$ utes after reinstitution of $\mathrm{CPB}$ (group $\mathrm{pH}\left[18^{\circ} \mathrm{C}\right.$ ).

Summary data for all animals are shown in Tables I through IV. For simplicity, statistical significance is designated in tables only for selected variables.

Control data. There were no significant differences in arterial or sagittal sinus $\mathrm{pH}$ or $\mathrm{PCO}_{2}$ values, mean arterial pressure, nasopharyngeal temperature, or cerebral oxygen extraction ratio between stage I and stage III. In the CTL $\left(18^{\circ} \mathrm{C}\right)$ animals after rewarming after 60 minutes of deep hypothermic CPB (stage III) there was a significant decrease in $\mathrm{CBF}$ compared with that in stage I. $\mathrm{CMRO}_{2}$ returned to baseline values in all animals regardless 
Table I. Summary data for all animals

\begin{tabular}{|c|c|c|c|c|c|c|c|c|}
\hline & \multicolumn{4}{|c|}{$18^{\circ} \mathrm{C}$} & \multicolumn{4}{|c|}{$14^{\circ} \mathrm{C}$} \\
\hline & $C T L$ & $\alpha$ & $p H$ & $p H \rightarrow \alpha$ & $C T L$ & $\alpha$ & $p H$ & $p H \rightarrow \alpha$ \\
\hline \multicolumn{9}{|c|}{$\mathrm{NPT}\left({ }^{\circ} \mathrm{C}\right)$} \\
\hline I & $37.4 \pm 0.1$ & $37.5 \pm 0.2$ & $37.0 \pm 0.2$ & $37.2 \pm 0.2$ & $37.4 \pm 0.6$ & $36.8 \pm 0.2$ & $37.1 \pm 0.2$ & $37.4 \pm 0.3$ \\
\hline II & $18.4 \pm 0.3$ & $18.6 \pm 0.2$ & $18.4 \pm 0.2$ & $18.1 \pm 0.1$ & $14.5 \pm 0.3$ & $13.6 \pm 0.3$ & $13.6 \pm 0.3$ & $14.3 \pm 0.3$ \\
\hline III & $36.9 \pm 0.3$ & $36.7 \pm 0.3$ & $36.6 \pm 0.3$ & $35.9 \pm 0.3$ & $35.8 \pm 0.1$ & $37.0 \pm 0.0$ & $36.1 \pm 0.1$ & $36.4 \pm 0.2$ \\
\hline \multicolumn{9}{|c|}{ Brain $\mathrm{T}\left({ }^{\circ} \mathrm{C}\right)$} \\
\hline I & $36.8 \pm 0.3$ & $37.1 \pm 0.5$ & $34.9 \pm 0.4^{*} \dagger$ & $37.1 \pm 0.3 \div$ & $37.9 \pm 0.5$ & $35.3 \pm 0.5^{*} \dagger$ & $36.3 \pm 0.6$ & $37.4 \pm 0.4$ \\
\hline $\mathrm{II}$ & $21.2 \pm 0.5$ & $21.2 \pm 0.6$ & $21.6 \pm 0.6$ & $19.3 \pm 0.4^{*}+7$ & $17.1 \pm 0.6$ & $17.8 \pm 1.2$ & $16.5 \pm 0.8$ & $17.6 \pm 1.1$ \\
\hline III & $34.4 \pm 0.4 \S$ & $33.8 \pm 0.8 \S$ & $31.7 \pm 0.6^{*}+\S$ & $32.9 \pm 0.6 \S$ & $33.6 \pm 1.2$ & $33.5 \pm 0.7$ & $33.2 \pm 1.0 \S$ & $33.4 \pm 0.9 \S$ \\
\hline \multicolumn{9}{|c|}{ MAP (mm $\mathrm{Hg})$} \\
\hline I & $62 \pm 8$ & $55 \pm 5$ & $46 \pm 6$ & $38 \pm 2$ & $33 \pm 1$ & $45 \pm 6$ & $46 \pm 2$ & $45 \pm 4$ \\
\hline II & $83 \pm 6$ & $69 \pm 5$ & $66 \pm 6$ & $61 \pm 4$ & $57 \pm 1$ & $65 \pm 8$ & $63 \pm 4$ & $57 \pm 4$ \\
\hline III & $70 \pm 9$ & $55 \pm 7$ & $53 \pm 8$ & $49 \pm 3$ & $45 \pm 5$ & $58 \pm 9$ & $57 \pm 4$ & $58 \pm 6$ \\
\hline \multicolumn{9}{|c|}{$\mathrm{HCT}(\%)$} \\
\hline I & $26 \pm 1$ & $26 \pm 1$ & $27 \pm 1$ & $26 \pm 1$ & $25 \pm 1$ & $26 \pm 1$ & $27 \pm 2$ & $27 \pm 0$ \\
\hline II & $26 \pm 1$ & $25 \pm 1$ & $27 \pm 1$ & $26 \pm 1$ & $25 \pm 2$ & $25 \pm 1$ & $27 \pm 2$ & $27 \pm 1$ \\
\hline III & $33 \pm 2 \S$ & $27 \pm 1^{*}$ & $28 \pm 1^{*}$ & $27 \pm 1^{*}$ & $30 \pm 1$ & $27 \pm 1$ & $26 \pm 2$ & $28 \pm 1$ \\
\hline
\end{tabular}

Values given as mean plus or minus standard error. $C T L$, Control; $\alpha$, alpha-stat; $p H, \mathrm{pH}$-stat; $p H \rightarrow \alpha, \mathrm{pH}$-stat switched to alpha-stat; $I, \mathrm{CPB}\left(37^{\circ} \mathrm{C}\right) ; I I, \mathrm{CPB}$ $\left(18^{\circ}\right.$ or $\left.14^{\circ} \mathrm{C}\right) ; I I I, \mathrm{CPB}\left(36^{\circ} \mathrm{C}\right) ; N P T$, nasopharyngeal temp; Brain T, cortical temp; $M A P$, mean arterial pressure; $H C T$, hematocrit.

$* p<0.05$, all groups versus control.

$\dagger p<0.05$, all groups versus $\alpha\left(18^{\circ} \mathrm{C}\right)$.

$\ddagger p<0.05$, all groups versus $\mathrm{pH}\left(18^{\circ} \mathrm{C}\right)$.

$\$ p<0.05$, stage III versus stage I.

Table II. Arterial and sagittal sinus blood gas data for animals cooled to $18^{\circ} \mathrm{C}$

\begin{tabular}{|c|c|c|c|c|}
\hline & $C T L$ & $\alpha$ & $p H$ & $p H \rightarrow \alpha$ \\
\hline \multicolumn{5}{|l|}{$\mathrm{apH}$} \\
\hline $\mathrm{I}$ & $7.40 \pm 0.01$ & $7.39 \pm 0.15$ & $7.41 \pm 0.01$ & $7.40 \pm 0.01$ \\
\hline II & $7.38 \pm 0.02$ & $7.34 \pm 0.13$ & $7.13 \pm 0.02 *+\ddagger(7.40 \pm 0.02)$ & $7.09 \pm 0.01 * \dagger(7.37 \pm 0.01) \rightarrow 7.35 \pm 0.01$ \\
\hline III & $7.41 \pm 0.01$ & $7.38 \pm 0.01$ & $7.40 \pm 0.01$ & $7.39 \pm 0.01$ \\
\hline \multicolumn{5}{|c|}{$\mathrm{PaCO}_{2}(\mathrm{~mm} \mathrm{Hg})$} \\
\hline I & $38 \pm 1$ & $37 \pm 1$ & $38 \pm 1$ & $38 \pm 1$ \\
\hline II & $40 \pm 2$ & $44 \pm 2$ & $83 \pm 7^{*}+1(36 \pm 2)$ & $93 \pm 3^{*} \dagger(37 \pm 1)->42 \pm 1$ \\
\hline III & $38 \pm 1$ & $37 \pm 1$ & $37 \pm 0$ & $39 \pm 1$ \\
\hline \multicolumn{5}{|l|}{$\mathrm{sspH}$} \\
\hline $\mathrm{I}$ & $7.29 \pm 0.01$ & $7.27 \pm 0.01$ & $7.27 \pm 0.02$ & $7.27 \pm 0.01$ \\
\hline II & $7.30 \pm 0.02$ & $7.25 \pm 0.01$ & $7.08 \pm 0.01 *+7$ & $7.05 \pm 0.01 \rightarrow 7.27 \pm 0.01 \S$ \\
\hline III & $7.32 \pm 0.02$ & $7.27 \pm 0.02$ & $7.23 \pm 0.02$ & $7.25 \pm 0.02$ \\
\hline \multicolumn{5}{|c|}{$\mathrm{PssCO}_{2}(\mathrm{~mm} \mathrm{Hg})$} \\
\hline I & $55 \pm 2$ & $55 \pm 1$ & $56 \pm 2$ & $56 \pm 1$ \\
\hline II & $50 \pm 2$ & $56 \pm 2$ & $95 \pm 2 *+1$ & $102 \pm 2 \rightarrow 53 \pm 1 \S$ \\
\hline III & $52 \pm 2$ & $56 \pm 5$ & $60 \pm 3$ & $57 \pm 2$ \\
\hline
\end{tabular}

Values given as mean plus or minus standard error. Temperature-uncorrected values given with temperature-corrected values in parentheses. $C T L$, Control (60 minutes deep hypothermic CPB); $\alpha$, alpha-stat; $p H$, pH-stat; $p H \rightarrow \alpha, \mathrm{pH}$-stat switched to alpha-stat; $I, \mathrm{CPB}\left(37^{\circ} \mathrm{C}\right) ; I I, \mathrm{CPB}\left(18^{\circ}\right.$ or $\left.14^{\circ} \mathrm{C}\right) ; I I I, \mathrm{CPB}$ $\left(36^{\circ} \mathrm{C}\right) ; a p H$, arterial $\mathrm{pH} ; s \mathrm{pH}$, sagittal sinus $\mathrm{pH} ; \mathrm{PSSCO}_{2}$, sagittal sinus carbon dioxide tension.

${ }^{*} p<0.05$, all groups versus control.

$\dagger p<0.05$, all groups versus $\alpha\left(18^{\circ} \mathrm{C}\right)$.

$\ddagger p<0.05$, all groups versus $\alpha\left(14^{\circ} \mathrm{C}\right)$.

$\$ p<0.05$, all groups versus $\mathrm{pH}\left(18^{\circ} \mathrm{C}\right)$.

of final cooling temperature $\left(18^{\circ}\right.$ or $\left.14^{\circ} \mathrm{C}\right)$ after rewarming. It is also interesting to note that $\mathrm{CMRO}_{2}$ returned to baseline values even though there was a significant decrease in cortical brain temperature in $\mathrm{CTL}\left(18^{\circ} \mathrm{C}\right)$ group animals at stage
III after rewarming compared with that in stage I. There was a significant increase in hematocrit concentration in the $\mathrm{CTL}\left(18^{\circ} \mathrm{C}\right)$ group at stage III compared with that at stage $\mathrm{I}$; however, this increase was not believed to be large enough to account for 
Table III. Arterial and sagittal sinus blood gas data for animals cooled to $14^{\circ} \mathrm{C}$

\begin{tabular}{|c|c|c|c|c|}
\hline & $C T L$ & $\alpha$ & $p H$ & $p H \rightarrow \alpha$ \\
\hline \multicolumn{5}{|l|}{ apH } \\
\hline I & $7.38 \pm 0.02$ & $7.39 \pm 0.02$ & $7.40 \pm 0.01$ & $7.38 \pm 0.01$ \\
\hline II & $7.40 \pm 0.03$ & $7.38 \pm 0.01$ & $6.95 \pm 0.03^{*} \div \div 8(7.29 \pm 0.01)$ & $7.01 \pm 0.01 * 4 \|(7.35 \pm 0.01) \rightarrow 7.38 \pm 0.02$ \\
\hline III & $7.43 \pm 0.02$ & $7.40 \pm 0.01$ & $7.41 \pm 0.02$ & $7.42 \pm 0.01$ \\
\hline \multicolumn{5}{|c|}{$\mathrm{PaCO}_{2}(\mathrm{~mm} \mathrm{Hg})$} \\
\hline I & $39 \pm 3$ & $40 \pm 2$ & $40 \pm 1$ & $39 \pm 1$ \\
\hline II & $39 \pm 4$ & $42 \pm 1$ & $133 \pm 8 *+\S(43 \pm 3)$ & $123 \pm 4^{*}+(41 \pm 1) \rightarrow 39 \pm 2$ \\
\hline III & $37 \pm 0$ & $37 \pm 1$ & $39 \pm 1$ & $38 \pm 1$ \\
\hline \multicolumn{5}{|l|}{ sspH } \\
\hline I & $7.26 \pm 0.02$ & $7.23 \pm 0.01$ & $7.27 \pm 0.01$ & $7.28 \pm 0.02$ \\
\hline II & $7.32 \pm 0.02$ & $7.30 \pm 0.01$ & $6.92 \pm 0.03^{*}+ \pm 8$ & $6.99 \pm 0.01 \rightarrow 7.27 \pm 0.01 \|$ \\
\hline III & $7.31 \pm 0.03$ & $7.27 \pm 0.00$ & $7.28 \pm 0.02$ & $7.29 \pm 0.01$ \\
\hline \multicolumn{5}{|c|}{$\mathrm{PssCO}_{2}(\mathrm{~mm} \mathrm{Hg})$} \\
\hline I & $61 \pm 3$ & $61 \pm 1$ & $56 \pm 1$ & $60 \pm 1$ \\
\hline II & $50 \pm 4$ & $50 \pm 2$ & $131 \pm 7^{*}+5 \S$ & $123 \pm 3 \rightarrow 53 \pm 2 \|$ \\
\hline III & $54 \pm 3$ & $52 \pm 2$ & $56 \pm 3$ & $57 \pm 3$ \\
\hline
\end{tabular}

Values given as mean plus or minus standard error. Temperature-uncorrected values given with temperature-corrected values in parentheses. CTL, Control ( 60 minutes deep hypothermic $\mathrm{CPB}) ; \alpha$, alpha-stat; $p H$, pH-stat; $p H \rightarrow \alpha$; pH-stat switched to alpha-stat; $I, \mathrm{CPB}\left(37^{\circ} \mathrm{C}\right) ; I I, \mathrm{CPB}\left(18^{\circ}\right.$ or $\left.14^{\circ} \mathrm{C}\right) ; I I I, \mathrm{CPB}$ $\left(36^{\circ} \mathrm{C}\right) ; a p H$, arterial $\mathrm{pH} ; s \mathrm{pH}$, sagittal sinus $\mathrm{pH}$; $\mathrm{PssCO}_{2}$, sagittal sinus carbon dioxide tension.

${ }^{*} p<0.05$, all groups versus control.

$\dagger p<0.05$, all groups versus $\alpha\left(18^{\circ} \mathrm{C}\right)$.

$\ddagger p<0.05$, all groups versus $\alpha\left(14^{\circ} \mathrm{C}\right)$.

$\S p<0.05$, all groups versus $\mathrm{pH}\left(18^{\circ} \mathrm{C}\right)$

$\| p<0.05$, all groups versus $\mathrm{pH}\left(14^{\circ} \mathrm{C}\right)$.

Table IV. Summary data for all animals

\begin{tabular}{|c|c|c|c|c|c|c|c|c|}
\hline & \multicolumn{4}{|c|}{$18^{\circ} \mathrm{C}$} & \multicolumn{4}{|c|}{$14^{\circ} \mathrm{C}$} \\
\hline & $C T L$ & $\alpha$ & $p H$ & $p H \rightarrow \alpha$ & $C T L$ & $\alpha$ & $p H$ & $p H \rightarrow \alpha$ \\
\hline \multicolumn{9}{|c|}{$\mathrm{CBF}(\mathrm{ml} / 100 \mathrm{gm} / \mathrm{min})$} \\
\hline I & $38.8 \pm 2.0$ & $36.0 \pm 2.3$ & $32.1 \pm 1.8^{*}$ & $30.4 \pm 0.8^{*} \dagger$ & $26.4 \pm 1.0$ & $32.4 \pm 2.5$ & $34.7 \pm 1.1^{*}$ & $31.4 \pm 1.4$ \\
\hline II & $35.8 \pm 2.0$ & $32.6 \pm 2.7$ & $27.4 \pm 2.1$ & $28.6 \pm 1.0$ & $26.0 \pm 1.9$ & $28.5 \pm 3.2$ & $33.7 \pm 1.8^{*}$ & $26.5 \pm 1.2$ \\
\hline III & $31.0 \pm 3.4 \ddagger$ & $30.2 \pm 2.0+$ & $23.9 \pm 1.0 \ddagger$ & $24.0 \pm 0.7^{*} \dagger$ & $22.1 \pm 0.8$ & $26.1 \pm 1.5 \$$ & $25.9 \pm 1.2 \ddagger$ & $27.3 \pm 1.1 \ddagger$ \\
\hline \multicolumn{9}{|c|}{$\mathrm{CMRO}_{2}(\mathrm{ml} / 100 \mathrm{gm} / \mathrm{min})$} \\
\hline I & $3.01 \pm 0.13$ & $3.06 \pm 0.13$ & $2.79 \pm 0.12$ & $2.65 \pm 0.10^{*} \frac{1}{\dagger}$ & $2.68 \pm 0.18$ & $2.52 \pm 0.13 \uparrow$ & $2.74 \pm 0.14$ & $2.71 \pm 0.10$ \\
\hline II & $1.19 \pm 0.08$ & $1.30 \pm 0.30$ & $1.05 \pm 0.06 \uparrow \S$ & $0.83 \pm 0.05^{*} \|$ & $0.94 \pm 0.17$ & $0.80 \pm 0.06 \dagger$ & $0.56 \pm 0.06^{*}+8 \|$ & $0.75 \pm 0.05 \%$ \\
\hline III & $2.97 \pm 0.21$ & $2.30 \pm 0.20 *+$ & $2.26 \pm 0.09^{*}+\S$ & $2.08 \pm 0.11^{*} \div$ & $2.66 \pm 0.14$ & $1.91 \pm 0.06^{*} \ddagger$ & $1.91 \pm 0.11^{*}$ 靸 & $2.35 \pm 0.08 \div \$ \pi$ \\
\hline \multicolumn{9}{|c|}{$\mathrm{CO}_{2} \mathrm{ER}(\%)$} \\
\hline $\mathrm{I}$ & $64.7 \pm 3.5$ & $68.3 \pm 2.1$ & $67.7 \pm 3.0$ & $69.0 \pm 2.2$ & $82.3 \pm 1.7$ & $64.6 \pm 4.9$ & $62.3 \pm 1.8$ & $65.4 \pm 1.1$ \\
\hline II & $25.9 \pm 2.1$ & $31.2 \pm 2.9$ & $25.9 \pm 1.8$ & $22.4 \pm 1.5$ & $27.3 \pm 4.9$ & $22.9 \pm 3.2$ & $12.5 \pm 1.4 * \dagger \S \|$ & $20.7 \pm 2.1 \pi$ \\
\hline III & $62.5 \pm 4.6$ & $66.2 \pm 3.9$ & $70.5 \pm 3.1$ & $65.7 \pm 2.4$ & $82.0 \pm 1.6$ & $58.4 \pm 4.8$ & $60.3 \pm 4.8$ & $62.8 \pm 1.9$ \\
\hline
\end{tabular}

Values given as mean plus or minus standard error. $C T L$, Control; $\alpha$, alpha-stat; $p H, \mathrm{pH}$-stat; $p H \rightarrow \alpha$, pH-stat switched to alpha-stat; $I, \mathrm{CPB}\left(37^{\circ} \mathrm{C}\right) ; I, \mathrm{CPB}$ $\left(18^{\circ}\right.$ or $\left.14^{\circ} \mathrm{C}\right) ; I I I, \mathrm{CPB}\left(36^{\circ} \mathrm{C}\right) ; \mathrm{CO}_{2} E R$, cerebral oxygen extraction ratio.

${ }^{*} p<0.05$, all groups versus control.

$\dagger p<0.05$, all groups versus $\alpha\left(18^{\circ} \mathrm{C}\right)$

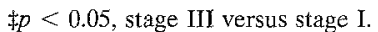

$\$ p<0.05$, all groups versus $\alpha\left(14^{\circ} \mathrm{C}\right)$.

$\| p<0.05$, all groups versus $\mathrm{pH}\left(18^{\circ} \mathrm{C}\right)$.

I $p<0.05$, all groups versus $\mathrm{pH}\left(14^{\circ} \mathrm{C}\right)$.

the return of $\mathrm{CMRO}_{2}$ to baseline levels in these animals.

Effects of cooling temperature on $\mathrm{CMRO}_{2}$. Cortical brain temperatures were significantly lower in those groups of animals cooled to $14^{\circ} \mathrm{C}$ versus those cooled to $18^{\circ} \mathrm{C}$. At stage II (Fig. 2), $\mathrm{CMRO}_{2}$ was significantly more suppressed at $14^{\circ} \mathrm{C}$ than at $18^{\circ} \mathrm{C}$. Regardless of the final cooling temperature and the amount of cerebral metabolic suppression at stage II, 60 minutes of DHCA resulted in a significant reduction in $\mathrm{CMRO}_{2}$ after rewarming compared with baseline values (Fig. 3). 


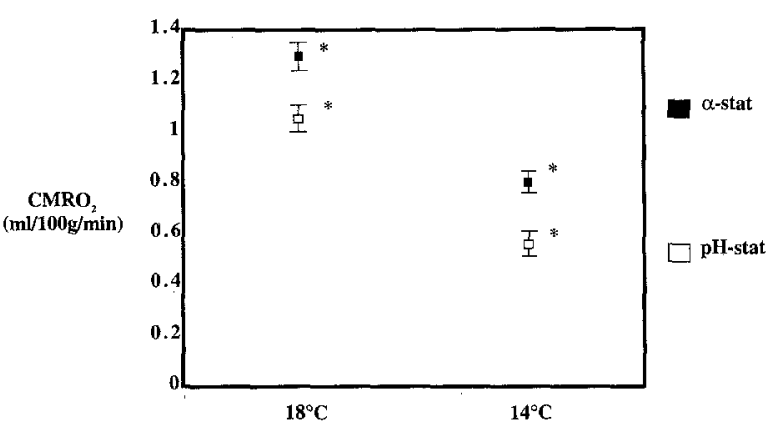

Fig. 2. $\mathrm{CMRO}_{2}$ measurements before DHCA at maximal cooling $\left(18^{\circ}\right.$ or $\left.14^{\circ} \mathrm{C}\right)$. $\mathrm{CMRO}_{2}$ is more suppressed at $14^{\circ} \mathrm{C}$ compared with value at $18^{\circ} \mathrm{C}$. At any given temperature $\left(18^{\circ}\right.$ or $\left.14^{\circ} \mathrm{C}\right)$, $\mathrm{pH}$-stat strategy provides better suppression of $\mathrm{CMRO}_{2}$. Mean data plus or minus standard error are shown. ${ }^{*} p<0.05$ versus all other groups at maximal cooling.

Effects of blood gas management (alpha-stat versus pH-stat) on $\mathrm{CMRO}_{2}$. There were no significant differences in cortical brain temperatures at stage II between the $\alpha\left(18^{\circ} \mathrm{C}\right)$ and $\mathrm{pH}\left(18^{\circ} \mathrm{C}\right)$ or $\alpha\left(14^{\circ} \mathrm{C}\right)$ and $\mathrm{pH}\left(14^{\circ} \mathrm{C}\right)$ groups. At any given temperature $\left(18^{\circ}\right.$ or $\left.14^{\circ} \mathrm{C}\right)$, pH-stat strategy provided significantly better suppression of $\mathrm{CMRO}_{2}$ at stage II compared with that with the use of alpha-stat strategy (Fig. 2). CBF decreased at stage III in groups $\alpha\left(18^{\circ} \mathrm{C}\right), \mathrm{pH}\left(18^{\circ} \mathrm{C}\right), \alpha\left(14^{\circ} \mathrm{C}\right)$, and $\mathrm{pH}\left(14^{\circ} \mathrm{C}\right) \mathrm{com}-$ pared with that at stage I. Cerebral oxygen extraction ratio at stage III was significantly decreased in the group $\mathrm{pH}\left(14^{\circ} \mathrm{C}\right)$ animals compared with that in the $\alpha\left(18^{\circ} \mathrm{C}\right), \mathrm{pH}\left(18^{\circ} \mathrm{C}\right)$, and $\alpha\left(14^{\circ} \mathrm{C}\right)$ groups.

Even though the use of $\mathrm{pH}$-stat strategy provided the least amount of $\mathrm{CMRO}_{2}$ at stage II, 60 minutes of DHCA resulted in a significant reduction of $\mathrm{CMRO}_{2}$ after rewarming compared with baseline values in the $\mathrm{pH}\left(18^{\circ} \mathrm{C}\right)$ and $\mathrm{pH}\left(14^{\circ} \mathrm{C}\right)$ group animals and in the $\alpha\left(18^{\circ} \mathrm{C}\right)$ and $\alpha\left(14^{\circ} \mathrm{C}\right)$ groups (Fig. $3)$. There were no significant differences in the amount of cerebral metabolic recovery at stage III between the $\alpha\left(18^{\circ} \mathrm{C}\right)$ and $\mathrm{pH}\left(18^{\circ} \mathrm{C}\right)$ or $\alpha\left(14^{\circ} \mathrm{C}\right)$ and $\mathrm{pH}\left(14^{\circ} \mathrm{C}\right)$ groups. There was, however, a significant reduction in $\mathrm{CMRO}_{2}$ at stage III in the $\alpha\left(14^{\circ} \mathrm{C}\right)$ and $\mathrm{pH}\left(14^{\circ} \mathrm{C}\right)$ groups compared with that in the $\mathrm{pH}\left(18^{\circ} \mathrm{C}\right)$ group (Fig. 3). This reduction in $\mathrm{CMRO}_{2}$ at stage III was not seen when the $\alpha\left(18^{\circ} \mathrm{C}\right)$ and $\alpha\left(14^{\circ} \mathrm{C}\right)$ groups were compared. These reductions in $\mathrm{CMRO}_{2}$ may be the result of the use of such profound hypothermia in those animals cooled to $14^{\circ} \mathrm{C}$. There was a significant difference in sagittal sinus $\mathrm{pH}$, venous carbon dioxide tension $\left(\mathrm{PvCO}_{2}\right)$,

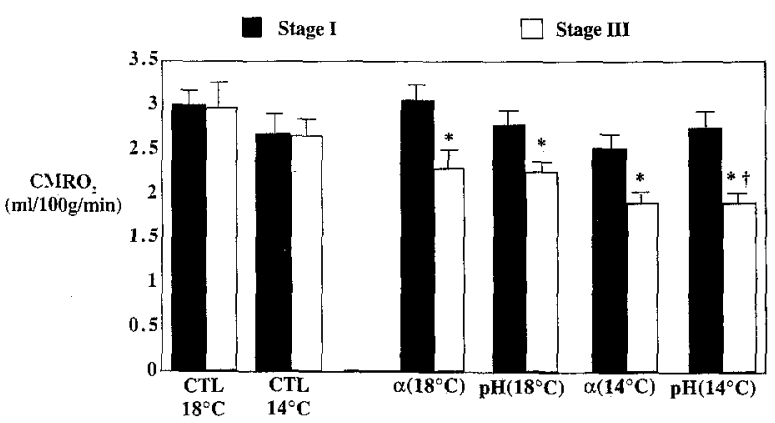

Fig. 3. $\mathrm{CMRO}_{2}$ measurements before and after deep hypothermic CPB or DHCA. Control (CTL) animals had almost complete return of $\mathrm{CMRO}_{2}$ to baseline values after deep hypothermic CPB. There was significant decrease in $\mathrm{CMRO}_{2}$ from stage I to stage III in all animals undergoing 60 minutes of DHCA. Mean data plus or minus standard error are shown. ${ }^{*} p<0.05$, stage III versus stage $\mathrm{I}$; $\uparrow p<0.05, \mathrm{pH}\left(14^{\circ} \mathrm{C}\right)$ versus $\mathrm{pH}\left(18^{\circ} \mathrm{C}\right) ; I$, $\mathrm{CPB}\left(37^{\circ} \mathrm{C}\right) ; I I I, \mathrm{CPB}\left(36^{\circ} \mathrm{C}\right)$ after rewarming.

and $\mathrm{PaCO}_{2}$ values in the $\mathrm{pH}\left(18^{\circ} \mathrm{C}\right)$ and $\mathrm{pH}\left(14^{\circ} \mathrm{C}\right)$ groups compared with those values in the $\alpha\left(18^{\circ} \mathrm{C}\right)$ and $\alpha\left(14^{\circ} \mathrm{C}\right)$ animals (Tables II and III). Animals in the pH-stat groups were profoundly acidotic with very low $\mathrm{pH}$ and high $\mathrm{PvCO}_{2}$ values, especially those animals cooled to $14^{\circ} \mathrm{C}$.

$\mathbf{p H} \rightarrow \boldsymbol{\alpha}$-stat data. There was a significant increase in sagittal sinus $\mathrm{pH}$ and a significant decrease in sagittal sinus $\mathrm{PvCO}_{2}$ at stage $\mathrm{II}$ in the $\mathrm{pH} \rightarrow \alpha\left(18^{\circ} \mathrm{C}\right)$ and $\mathrm{pH} \rightarrow \alpha\left(14^{\circ} \mathrm{C}\right)$ animals compared with respective values in the $\mathrm{pH}\left(18^{\circ} \mathrm{C}\right)$ and $\mathrm{pH}\left(14^{\circ} \mathrm{C}\right)$ groups. The cortical brain temperature and $\mathrm{CMRO}_{2}$ at stage II were significantly lower in the $\mathrm{pH} \rightarrow \alpha\left(18^{\circ} \mathrm{C}\right)$ group than these values in the $\alpha\left(18^{\circ} \mathrm{C}\right)$ and $\mathrm{pH}\left(18^{\circ} \mathrm{C}\right)$ group animals. These differences in cortical brain temperature and $\mathrm{CMRO}_{2}$ at stage II did not occur in the $\mathrm{pH} \rightarrow \alpha\left(14^{\circ} \mathrm{C}\right)$ animals. CBF decreased at stage III in the $\mathrm{pH} \rightarrow \alpha\left(18^{\circ} \mathrm{C}\right)$ and $\mathrm{pH} \rightarrow \alpha\left(14^{\circ} \mathrm{C}\right)$ groups compared with values at stage I. $\mathrm{CMRO}_{2}$ was significantly increased at stage III in the $\mathrm{pH} \rightarrow \alpha\left(14^{\circ} \mathrm{C}\right)$ animals compared with this value in the $\alpha\left(14^{\circ} \mathrm{C}\right)$ and $\mathrm{pH}\left(14^{\circ} \mathrm{C}\right)$ groups (Fig. 4). There was no significant difference in $\mathrm{CMRO}_{2}$ at stage III in the $\mathrm{pH} \rightarrow \alpha\left(18^{\circ} \mathrm{C}\right)$ group compared with the value in the $\alpha\left(18^{\circ} \mathrm{C}\right)$ and $\mathrm{pH}\left(18^{\circ} \mathrm{C}\right)$ group animals. Again, 60 minutes of DHCA resulted in decreased $\mathrm{CMRO}_{2}$ after rewarming compared with baseline values in both $\mathrm{pH} \rightarrow \alpha\left(18^{\circ} \mathrm{C}\right)$ and $\mathrm{pH} \rightarrow \alpha\left(14^{\circ} \mathrm{C}\right)$ groups.

The baseline cortical brain temperatures were significantly lower in the $\mathrm{pH}\left(18^{\circ} \mathrm{C}\right)$ and $\alpha\left(14^{\circ} \mathrm{C}\right)$ 
groups; however, this was not believed to be of physiologic importance. The cortical brain temperatures at stage III decreased significantly in the $\alpha\left(18^{\circ} \mathrm{C}\right), \mathrm{pH}\left(18^{\circ} \mathrm{C}\right), \mathrm{pH} \rightarrow \alpha\left(18^{\circ} \mathrm{C}\right), \mathrm{pH} \rightarrow \alpha\left(14^{\circ} \mathrm{C}\right)$, and $\mathrm{pH}\left(14^{\circ} \mathrm{C}\right)$ groups compared with those at stage I. A similar decrease in brain temperature after rewarming occurred in the $\mathrm{CTL}\left(18^{\circ} \mathrm{C}\right)$ animals, and this did not seem to affect the recovery of $\mathrm{CMRO}_{2}$ to baseline values. Baseline $\mathrm{CMRO}_{2}$ values in the $\mathrm{pH} \rightarrow \alpha\left(18^{\circ} \mathrm{C}\right)$ and $\alpha\left(14^{\circ} \mathrm{C}\right)$ animals were significantly lower compared with those in the $\operatorname{CTL}\left(18^{\circ} \mathrm{C}\right)$ and $\alpha\left(18^{\circ} \mathrm{C}\right)$ groups and in the $\alpha\left(18^{\circ} \mathrm{C}\right)$ group, respectively. A strict study protocol was adhered to for each animal in regard to anesthesia and instrumentation, which makes these unlikely explanations for these differences. The data were analyzed with use of the raw change in $\mathrm{CMRO}_{2}$ comparing stage I with stage III values within groups; therefore, these baseline differences should not interfere with data analysis.

\section{Discussion}

The prevalence of neuropsychologic injury occurring after DHCA for the repair of complex congenital heart defects may be as high as $25 \%$. $^{2}$ The principal method of cerebral protection during DHCA is the use of hypothermia. Cerebral metabolism decreases exponentially with reductions in temperature. ${ }^{5}$ A reduction in $\mathrm{CMRO}_{2}$ decreases the depletion rate of high-energy phosphate compounds and the development of intracellular acidosis that occurs during circulatory arrest. ${ }^{4,16,17}$ These findings emphasize the importance of the uniform delivery of cold perfusate to the brain so that a homogeneous reduction in cerebral temperature and $\mathrm{CMRO}_{2}$ occurs at the start of DHCA. Cerebral metabolic recovery after DHCA may be enhanced by strategies that provide the least amount of cerebral metabolism before DHCA. The use of lower cooling temperatures and use of the cerebral vasodilator $\mathrm{CO}_{2}$ (pH-stat blood gas strategy) are two strategies that by themselves or in combination may result in improved cerebral metabolic recovery after a period of DHCA.

This study demonstrates in this model of neonatal CPB that $\mathrm{CMRO}_{2}$ is more suppressed at $14^{\circ} \mathrm{C}$ than at $18^{\circ} \mathrm{C}$. At any given temperature, $18^{\circ}$ or $14^{\circ} \mathrm{C}$, $\mathrm{CMRO}_{2}$ is better suppressed in those animals cooled with pH-stat blood gas management (Fig. 2). Exposure to 60 minutes of DHCA, however, resulted in a significant decrease in $\mathrm{CMRO}_{2}$ from

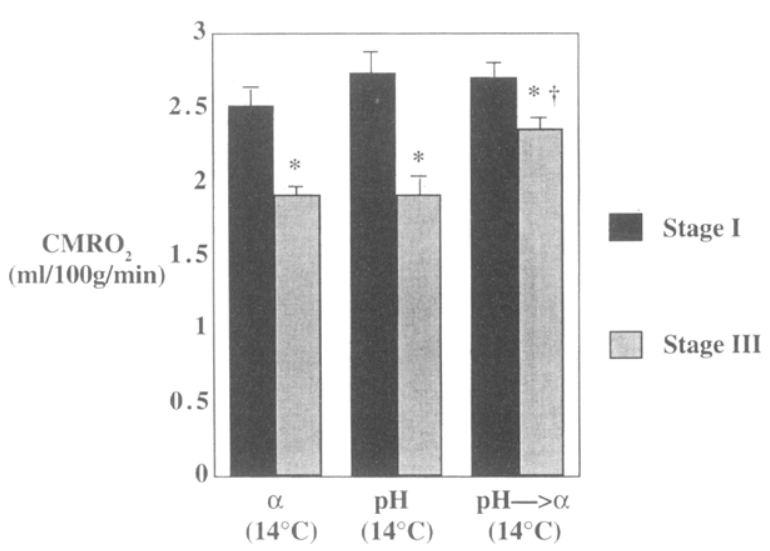

Fig. 4. $\mathrm{CMRO}_{2}$ measurements before and after DHCA in those animals cooled to $14^{\circ} \mathrm{C}$. There was significant decrease in $\mathrm{CMRO}_{2}$ from stage I to stage III in all animals. At stage III, there was significant decrease in $\mathrm{CMRO}_{2}$ in alpha-stat and $\mathrm{pH}$-stat groups compared with values in $\mathrm{pH} \rightarrow \alpha$-stat group animals. Mean data plus or minus standard error are given. ${ }^{*} p<0.05$, stage III versus stage I; $\nmid p<0.05, \mathrm{pH} \rightarrow \alpha$-stat versus alpha-stat and pH-stat; $I$, CPB $\left(37^{\circ} \mathrm{C}\right) ; I I I, \mathrm{CPB}\left(36^{\circ} \mathrm{C}\right)$ after rewarming.

baseline values after rewarming regardless of blood gas strategy or final cooling temperature (Fig. 3).

Possible mechanisms for the impairment in cerebral metabolic recovery after DHCA include inadequate rewarming of the brain after profound hypothermia, continued consumption of oxygen by the brain during the arrest period, and further reductions in intracellular $\mathrm{pH}$ value during the arrest period. The almost $100 \%$ recovery of $\mathrm{CMRO}_{2}$ seen in control animals after prolonged exposure to profound hypothermia $\left(14^{\circ}\right.$ or $\left.18^{\circ} \mathrm{C}\right)$, measured at statistically similar rewarming temperatures to those in the experimental groups, makes inadequate rewarming of the brain an unlikely mechanism (Fig. 3).

This study demonstrates that even though the use of $\mathrm{pH}$-stat at more profound hypothermic temperatures $\left(14^{\circ} \mathrm{C}\right)$ provides the least amount of cerebral metabolism, the brain continues to consume oxygen before and during the period of DHCA. The presence of continued cerebral metabolism at the start of DHCA has been shown to occur clinically and in other experimental models of neonatal $\mathrm{CPB}^{5,18,19}$ and may in part explain the impairment in $\mathrm{CMRO}_{2}$ after DHCA. Recent investigations have demonstrated that 1 hour of arrest, even at deep hypothermic temperatures, reduces the metabolic reserve of the brain. Strategies such as topical cooling of the brain, intermittent perfusion, and trickle flow may 
help prevent further cerebral injury from occurring during the arrest time. ${ }^{12,20}$

Cerebral metabolic recovery was significantly reduced in those animals cooled with $\mathrm{pH}$-stat to $14^{\circ} \mathrm{C}$ compared with that in the $\mathrm{pH}\left(18^{\circ} \mathrm{C}\right)$ group. This difference was not seen in those groups cooled with alpha-stat strategy. At the start of DHCA, the $\mathrm{pH}$-stat animals were profoundly acidotic with very low arterial and sagittal sinus $\mathrm{pH}$ values and high $\mathrm{PvCO}_{2}$ values, especially those cooled to $14^{\circ} \mathrm{C}$ (pH $6.95 \pm 0.03, \mathrm{pH} 6.92 \pm 0.03$, and $131 \pm 7 \mathrm{~mm} \mathrm{Hg}$, respectively). This degree of acidosis preceding DHCA may make the brain less tolerant of prolonged ischemia despite the degree of metabolic suppression. A recent investigation has demonstrated that if a period of arrest is used in addition to $\mathrm{pH}$-stat strategy, a further reduction occurs in intracellular $\mathrm{pH} .{ }^{11}$ The resultant severe cerebral acidosis may delay or prevent full brain recovery. Lower intracellular $\mathrm{pH}$ also results in an impairment in cellular enzymatic function. ${ }^{21}$

From the latter findings, a switch strategy was devised. pH-stat strategy would be used during initial cooling to provide maximal cerebral metabolic suppression. A switch to alpha-stat strategy would follow just before the period of arrest to decrease the severe acid debt that occurs at profound hypothermia with the use of $\mathrm{pH}$-stat. The sagittal sinus $\mathrm{pH}$ and $\mathrm{PvCO}_{2}$ values were not significantly different after the use of the switch strategy compared with those in the animals cooled to $14^{\circ} \mathrm{C}$ with alpha-stat strategy alone. The switch strategy provided significantly better recovery of cerebral metabolism after DHCA compared with recovery with either strategy used alone ( $\mathrm{pH}$-stat or alphastat) in those animals cooled to $14^{\circ} \mathrm{C}$ (Fig. 4). However, an impairment in cerebral metabolic recovery after 60 minutes of DHCA still occurred. When the switch strategy was used during cooling to $18^{\circ} \mathrm{C}$, there was no difference in cerebral metabolic recovery after DHCA compared with the recovery with either strategy used alone. This latter finding may be explained by the fact that the acid debt accrued with the use of $\mathrm{pH}$-stat at $18^{\circ} \mathrm{C}$ is not as large; therefore, the addition of a 60 -minute period of DHCA may not be as deleterious.

There are several limitations to this study. The cerebral metabolic response patterns demonstrated in these animals are acute changes only. However, recent investigations have demonstrated that DHCA is associated with an impairment in longterm neurologic outcome that may be related to the pattern of cerebral metabolic recovery seen in the immediate post-CPB period. ${ }^{22}$ The measurements of $\mathrm{CBF}$ are global and do not provide information about regional blood flow. However, this method of CBF measurement was chosen to parallel methods used in patients at this institution to make results produced in the laboratory setting clinically applicable. The brains were not examined histologically or on a molecular level. Further studies will need to be done to help understand these acute changes in cerebral metabolism on a molecular level.

In summary, this study demonstrates that $\mathrm{CMRO}_{2}$ is more suppressed at $14^{\circ} \mathrm{C}$ than at $18^{\circ} \mathrm{C}$. $\mathrm{pH}$-stat strategy provides better suppression of cerebral metabolism at any given temperature $\left(14^{\circ}\right.$ and $18^{\circ} \mathrm{C}$ ). The use of $\mathrm{pH}$-stat strategy during initial cooling to $14^{\circ} \mathrm{C}$ followed by a switch to alpha-stat strategy just before arrest results in better recovery of cerebral metabolism after DHCA compared with the recovery with either strategy used alone. Regardless of degree of cooling and blood gas strategy used, 60 minutes of DHCA results in an impairment in the recovery of cerebral metabolism after rewarming. The appropriateness of a blood gas strategy depends on many factors including the degree of hypothermia, pump flow rate, the use of DHCA, and the presence of anatomic variants (large aortopulmonary shunts). In situations in which inefficient cooling may occur because of anatomic variants or in which a prolonged use of DHCA is anticipated, the use of lower cooling temperatures and the cerebrovasodilation of $\mathrm{CO}_{2}$ may be beneficial. Once the patient is cool, however, a switch to alpha-stat strategy may help preserve intracellular $\mathrm{pH}$ by reducing the amount of postarrest cerebral acidosis.

\section{REFERENCES}

1. Ferry PC. Neurologic sequelae of cardiac surgery in children. Am J Dis Child 1987;141:309-12.

2. Ferry PC. Neurologic sequelae of open-heart surgery in children: an "irritating question." Am J Dis Child 1990;144:369-73.

3. Newburger JW, Jonas RA, Wernovsky G, et al. A comparison of the perioperative neurologic effects of hypothermic circulatory arrest versus low-flow cardiopulmonary bypass in infant heart surgery. $\mathrm{N}$ Engl J Med 1993;329:1057-64.

4. Norwood WI, Norwood CR, Ingwall JS, et al. Hypothermic circulatory arrest: 31-phosphorus nuclear magnetic resonance of isolated perfused neonatal rat brain. J Thorac Cardiovasc Surg 1979;78:823-30. 
5. Greeley WJ, Kern FH, Ungerleider RM, et al. The effect of hypothermic cardiopulmonary bypass and total circulatory arrest on cerebral metabolism in neonates, infants, and children. J THORAC CARDIOVASC SURG 1991;101:783-94.

6. Murkin JM, Farrar JK, Tweed WA, et al. Cerebral autoregulation and flow/metabolic coupling during cardiopulmonary bypass. Anesth Analg 1987;66:82532.

7. Prough DS, Stump DA, Roy RC, et al. Response of cerebral blood flow to changes in carbon dioxide tension during hypothermic cardiopulmonary bypass. Anesthesiology 1986;64:576-81.

8. Govier AV, Reves JG, McKay RD, et al. Factors and their influence on regional cerebral blood flow during nonpulsatile cardiopulmonary bypass. Ann Thorac Surg 1984;38:592-600.

9. Kern FH, Ungerleider RM, Quill TJ, et al. Cerebral blood flow response to changes in arterial carbon dioxide tension during hypothermic cardiopulmonary bypass in children. J THorac Cardiovasc Surg 1991; 101:618-22.

10. Rahn H, Reeves BR, Howell BJ. Hydrogen ion regulation, temperature, and evolution: the $1975 \mathrm{~J}$. Burns Amberson lecture. Am Rev Respir Dis 1975;112:16572.

11. Watanabe T, Miura M, Orita $H$, et al. Brain tissue $\mathrm{pH}$, oxygen tension, and carbon dioxide tension in profoundly hypothermic cardiopulmonary bypass: pulsatile assistance for circulatory arrest, low-flow perfusion, and moderate-flow perfusion. J THORAC Cardiovasc Surg 1990;100:274-80.

12. Mault IR, Ohtake S, Klingensmith ME, et al. Cerebral metabolism and circulatory arrest: effects of duration and strategies for protection. Ann Thorac Surg 1993;55:57-64.

13. Spahn DR, Quill TJ, Hu WC, et al. Validation of $133 \mathrm{X}$ e clearance as a cerebral blood flow measure- ment technique during cardiopulmonary bypass. $\mathrm{J}$ Cereb Blood Flow Metab 1992;12:155-61.

14. Oleson J, Paulson OB, Lassen NA. Regional cerebral blood flow in man determined by the initial slope of the clearance of intraarterially injected $133 \mathrm{Xe}$. Stroke 1971;2:519-25.

15. Chen RYZ, Fan FC, Kim S, et al. Tissue-blood partition coefficient for xenon: temperature and hematocrit dependence. J Appl Physiol 1980;49:178-83.

16. Stocker F, Hershkowitz N, Bossi E, et al. Cerebral metabolic studies in situ by 31P-nuclear magnetic resonance after hypothermic circulatory arrest. Pediatr Res 1986;20:867-71.

17. Chopp M, Knight R, Tidwell CD. The metabolic effects of moderate hypothermia on global cerebral ischemia and recirculation in the cat: comparison to monothermia and hypothermia. J Cereb Blood Flow Metab 1989;9:133-40.

18. Aoki M, Nomura F, Stromski ME, et al. Effects of $\mathrm{pH}$ on brain energetics after hypothermic circulatory arrest. Ann Thorac Surg 1993;55:1093-2103.

19. Mezrow CK, Midulla PS, Sadeghi AM, et al. Evaluation of cerebral metabolism and quantitative electroencephalography after hypothermic circulatory arrest and low-llow cardiopulmonary bypass at different temperatures. J THORAC CARDIOVASC SURG 1994;107: 1006-19.

20. Swain JA, McDonald TJ, Griffith PK, et al. Low flow hypothermic cardiopulmonary bypass protects the brain. J ThoraC CARDIOvasC Surg 1991;102:76-84.

21. Somero GN, White FN. Enzymatic consequences under alpha stat regulation. In: Rahn $\mathrm{H}$, Prakash $\mathrm{O}$, eds. Acid-base regulation and body temperature. Boston: Nijhoff, 1985:55-80.

22. Croughwell ND, Newman MF, Blumenthal JA, et al. Jugular venous saturation and cerebral arterial venous difference predict cognitive dysfunction after cardiac surgery. Circulation 1993;88(Suppl):1289. 